\title{
INDUCTION AND EXPLANATORY DEFINITIONS IN MATHEMATICS
}

\author{
ELLEN LEHET
}

\begin{abstract}
In this paper, I argue that there are cases of explanatory induction in mathematics. To do so, I first introduce the notion of explanatory definition in the context of mathematical explanation. A large part of the paper is dedicated to introducing and analyzing this notion of explanatory definition and the role it plays in mathematics. After doing so, I discuss a particular inductive definition in advanced mathematics $-C W$-complexes - and argue that it is explanatory. With this, we see that there are cases of explanatory induction.

Philosophy of Mathematics and Explanation and Mathematical Induction and Mathematical Definition and Mathematical Practice
\end{abstract}

\section{INTRODUCTION}

The question of whether mathematical induction is explanatory has proven to be controversial. A lot of the discussion has relied on people's intuitions about proofs by induction. It is important to realize, however, that mathematical induction is not used solely for proofs, but is also used for mathematical definitions. These cases of inductive definition provide another way in which induction might be explanatory. With an account of explanatory definition, we are able to evaluate inductive definitions and will see that they are (at least in some cases) explanatory. The objective of this paper is to introduce the notion of explanatory definition, and to demonstrate that some inductive definitions are explanatory.

In section 2, I will discuss the literature relating to explanation and mathematical induction. I will focus on Marc Lange's argument that induction is not explanatory, and will discuss 
two established criticisms of his argument. These criticisms will be used to motivate my own account of explanatory definition. In section 3, I will introduce the notion of explanatory definition, provide an illustrative example, and will analyze this account of explanatory definition. And in section 4, I argue that some instances of inductive definition are explanatory. Thus, we will see that my account of explanatory definitions is a substantial one and that induction is, in this sense, explanatory. In section 5, I make some concluding remarks.

\section{BaCkground: Marc LAnge}

Lange (2009) is titled "Why proofs by mathematical induction are generally not explanatory". This title suggests that Lange intends to show only that induction is "generally" not explanatory so that there may be exceptions - i.e., cases where induction is explanatory. But Lange's actual argument shows that, at least in most cases, inductive explanation turns out to be circular 1

Lange's argument turns on the fact that mathematical induction can be formulated in several different ways. The standard formulation is as follows:

PMI:: If $p(1)$ holds and for all $k \in \mathbb{N}, p(k) \Longrightarrow p(k+1)$, then $p(n)$ holds for all $n \in \mathbb{N}$.

But there's another way induction can be formulated:

ALT:: If $p(5)$ holds, and for all $k \in \mathbb{N}, p(k) \Longrightarrow p(k+1)$, and for all $k>1, p(k) \Longrightarrow$ $p(k-1)$, then $p(n)$ holds for all $n \in \mathbb{N}$.

\footnotetext{
${ }^{1}$ It should be noted that in Lange (2017), a revised account of the explanatory power of inductive proofs is presented. A reader particularly interested in explanatory proofs should refer to this work, but since I am only using criticisms of the Lange (2009) argument to motivate my own account I will not go into details about Lange's revisions here.
} 
These are both fine ways of formulating mathematical induction, but Lange's argument suggests that the alternative formulation prevents induction from being explanatory $2^{2}$ His argument goes as follows:

(1) Explanations cannot be circular.

(2) ALT is an equivalent formulation of mathematical induction to PMI.

(3) If $\mathbf{P M I}$ is used to show that for all $n \in \mathbb{N}, p(n)$ holds, then the fact that $p(1)$ holds helps to explain the fact that $p(5)$ holds.

(4) If ALT is used to show that for all $n \in \mathbb{N}, p(n)$ holds, then the fact that $p(5)$ holds helps to explain the fact that $p(1)$ holds.

(5) If mathematical induction is explanatory, then $p(1)$ explains $p(5)$ and $p(5)$ explains $p(1)$ and so the explanation is circular. $[2,3,4]$

(6) Thus, mathematical induction cannot be explanatory. $[1,5]$

Over the last decade, there have been many responses to this argument, but there are two specific criticisms that I will focus on. The first claims that Lange has misconstrued the way in which induction is explanatory. The second claims that Lange drastically oversimplifies the way that explanation is used in mathematics. In this paper, I will not provide new criticisms of Lange's argument, but will instead use these two criticisms to motivate my view about the potential explanatoriness of inductive definitions. Let me first summarize the basic ideas behind these criticisms.

The first criticism claims that Lange has misunderstood the way in which induction is used in mathematics and how that use can be connected to explanation. Lange's argument

${ }^{2}$ Lange takes these two formulations to be equivalent in the sense that both characterize induction equally well. This is a point that Baker (2010) objects to by pointing out that a case can be made for the claim that PMI has significant theoretical advantages. 
is deliberately vague about the nature of mathematical explanation. The hope is that the argument will apply to any reasonable account of explanation. So, Lange's only stipulation is that explanation cannot be circular. His goal is to show that, no matter which account of explanation you accept, induction will not be explanatory.

The criticism is that Lange has assumed more about explanation than he suggests $3^{3}$ Lange relies on the assumption that, if induction is explanatory, then it is explanatory when used in proofs by induction. So he is assuming that the core of mathematical explanation is explanatory

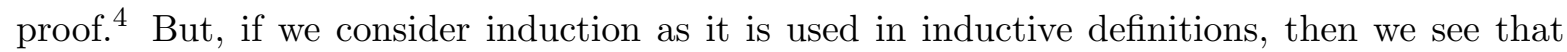
there are cases of explanatory induction. It is worth noting that mathematicians often describe definitions and constructions as explanatory:5 I will unpack the idea that some inductive definitions may be explanatory more in the next section.

The second criticism, which is closely related to the first, is that Lange oversimplifies the actual uses of induction in mathematics. Induction is not solely used for proving theorems about the properties of natural numbers. John Baldwin, for instance, has made this point about the use of induction in Henkin's proof of completeness (Baldwin $(2017))$. Here, induction is applied to more advanced mathematics, and it is used in a constructive way, one which seems to be more informative than the simple induction of Lange's argument. According to Baldwin,

\footnotetext{
${ }^{3}$ Others have made similar remarks. Baker (2010) points out that Lange seems to be relying on another assumption. Namely, the assumption that these two ways of formulating mathematical induction are theoretically equivalent. Baker goes on to argue that given some common assumptions about what we should expect from an account of explanation, these two are not equivalent. In particular, PMI has some explanatory virtues that Alt lacks. Hoeltje \& Schnieder \& Steinberg (2013) also makes similar comments. A related criticism is pointed out in Baldwin (2016), and states that Lange's argument does not only apply to proofs by induction, but also applies to universally axiomatized propositions. This is problematic since it seems that there are some such propositions that are, in fact, explanatory.

${ }^{4}$ To be fair, most of the existing literature on explanation within mathematics solely deals with explanatory proofs. Some exceptions include Hafner \& Mancosu (2005), Tappenden (2008a), and Tappenden (2008b), which have pointed out that mathematicians use the term explanation more broadly.

${ }^{5}$ Again this is also pointed out in Hafner \& Mancosu (2005), Tappenden (2008a), and Tappenden (2008b).
} 
the most interesting and deep uses of mathematical induction are ones like this, ones which appear in more abstract and advanced mathematics. Baldwin says:

There are too many investigations of 'explanation' to list that center around the extremely elementary uses of mathematical induction. But in more advanced mathematics the main use of induction is as [a] proof tool to study objects defined by generalized inductive definition. This includes not only such algebraic constructions as the closure of a set to a subgroup or in a logic, the set of formulas in logic or theorems of a theory, but constructions as in the Henkin proof: truth in a structure, completing a theory and fulfilling the witness property (Baldwin (2017), page 6).

The point here is that, in advanced mathematics, induction can give us a way of thinking about advanced mathematical concepts, ones of more complexity than the natural numbers. In these cases induction provides clarity for thinking and reasoning about these advanced concepts. Thus, induction is not used only to reason about simple structures like the natural numbers, but is also used to make more advanced and complex structures simpler.

In short, Lange's argument appeals only to the simplest use of induction in mathematics namely, its use for proving results about the natural numbers. For this reason, it is unclear whether his argument pulls any weight against more advanced uses of induction. When we consider inductive definitions in more advanced mathematics, the notion of induction is broadened so that it is no longer adequately characterized by Lange's formulation. Moreover, if it is correct that these more complex inductive definitions improve our ability to study abstract structures, then the question of the explanatoriness of these definitions themselves naturally 
arises - are the definitions themselves useful because they have some sort of explanatory value?

In my view, the answer to this question is yes, but I will wait until section 5 to explicitly argue for this claim.

In the next section, motivated by these criticisms, I will introduce the notion of explanatory definition. This notion will help to account for some of the ways that induction is used in advanced mathematics. Inductive definitions are one way that induction is used within mathematics and the notion of explanatory definition will allow us to see that this use of induction is (at least in some cases) explanatory. I will then focus on a particular, more advanced, inductive construction - i.e., $C W$-complexes — and I will argue that the inductive definition of these $C W$-complexes counts as an explanatory definition. In doing so, I will demonstrate the existence of explanatory induction.

\section{Explanatory Definitions}

Before presenting my account of explanatory definition, I need to highlight an important distinction between explanation in mathematics and explanation in the sciences. The relevant difference between mathematics and the sciences — i.e., the one that accounts for this difference in kinds of explanations — results from a difference in subject matter. The subject matter of the sciences is physical phenomena, but the subject matter of mathematics is much less concrete. In mathematics we study abstract concepts and constructions that for the most part cannot be found in the physical world. So, the scientist is given phenomena that is in need of explanation whereas the mathematician often needs to "construct" their objects of study. In

this way, the subject matter of the sciences is more accessible to us than that of mathematics ${ }^{6}$ that mathematics or mathematical objects are not the kinds of things that we can know by acquaintance. 
The mathematician needs to do a different kind of work in order to become familiar with and conjecture about their objects of study. So the mathematician must provide an explanation of the very nature of their objects of study before they can go about proving results.

Let me further motivate this difference in subject matter by comparing two examples - one in science and one in mathematics. A scientist can observe that two magnets are attracted to each other and wonder why this is the case. So they already have access to the relevant phenomena, and can immediately search for an explanatory theory of this phenomena. A mathematician studying group theory, cannot ask deep questions about groups before they have a good idea of the nature of groups. This is not something that can be obtained by observable phenomena as with the case of magnets. So, the mathematician must have some kind of explanation of the nature of groups before they can ask the kinds of theoretical whyquestions that scientists ask almost immediately. This is where explanatory definitions are introduced — that is, an explanatory definition provides a way of making sense of mathematical objects so that theories can progress and develop.

The failure to recognize this distinction between mathematics and the sciences is what has resulted in the focus on explanatory proof. By taking the mathematical analogue of scientific phenomena to be theorems, we see that it is natural to think of proofs as the potential providers of explanation in mathematics. This, however, overlooks some common why-questions that can arise. For instance, why a given definition is the right one. In Tappenden (2008a), Jamie Tappenden motivates his account of definition choice in mathematics by pointing out that mathematicians often ask questions about which definitions are "right" or "correct" and these questions influence the direction of mathematical research. In mathematics it is valuable to 
look for new definitions for already established and developed concepts. Doing so allows for new perspectives and provides ways of comparing different areas of mathematics. Thus, some definitions can be privileged over others and when this occurs it is often because the preferred definition is in some way more explanatory. This appreciation of new and different definitions is important for mathematical progress, but is less common in the sciences 7

Tappenden recognizes the epistemic significance of definitions in mathematics. He accepts what he calls the "Port Royal principle", a principle taken from Arnauld \& Nicole (1683) and stated as follows:

Nothing is more important in science than classifying and defining well... [though]

it depends much more on our knowledge of the subject matter being discussed than on the rules of logic (Tappenden (2008a), pg. 273).

This principle guides Tappenden in his discussion of the philosophical significance of definitions.

Given it, he goes on to develop a connection between the "identification of core properties and mathematical fecundity" (Tappenden $(2008 \mathrm{~b})$, pg. 293). It is this connection that he thinks accounts for the epistemic significance of definition choice. The "identification of core properties" provides insight by increasing our familiarity with the mathematical object; in turn, having this kind of insight allows for successful mathematical development.

The philosophical significance and epistemic value of definitions is something that an account of explanatory definition will capture, at least in part. I define this notion as follows:

\footnotetext{
${ }^{7}$ Note that though I claim that this practice is less common in the sciences, I do not mean to suggest that it does not occur at all. In fact, there are plenty of instances in the history of science that a change in standard definition occurs. It is more commonly the case, however, that scientific changes occur as a result of theoretical changes. That is, a change in theory inspires or results in a change in definition. In mathematics, this is not what occurs, but instead the theory remains the same and the definition changes to illuminate some evasive feature of the theory.
} 
Explanatory definition:: A mathematical definition is explanatory if it makes the mathematical concept being defined more accessible - i.e., it explains some feature or property of the relevant concept.

This notion of explanatory definition serves to supplement, but not to counter, the notion of explanatory proof in order to form a more complete account of mathematical explanation 8 It is important to note that the term "explanation" has been used in variety of ways in the philosophical literature. In the context of explanation in mathematics and the sciences, it is most commonly used to mean answers to why-questions.9 The way I am using it here is not in this sense. Instead, I am using it in the sense of illumination - an explanation is something that illuminates (or in my terms, makes more accessible). This may be criticized as a merely pedagogical sense of explanation, but such a criticism would misunderstand the nature of mathematical knowledge and progress ${ }^{10}$ As discussed above, methods and explanations that illuminate the subject matter of mathematics are important for mathematical knowledge in more than just a pedagogical sense. Now, to see that there are explanatory definitions, I will provide a concrete example.

Consider the derivative. William Thurston has pointed out that there are many different ways of defining the derivative ${ }^{11}$ On the surface, the sheer number of different definitions raises the question of which we should prefer. There is not one clear and uniform answer to

\footnotetext{
${ }^{8}$ I am not suggesting that the combination of explanatory definitions and explanatory proofs provides a comprehensive account of mathematical explanation. I would like to leave open the possibility that there are even still other forms of explanation in mathematics.

${ }^{9}$ In particular, the literature on explanatory proofs, and Lange's argument discussed above, takes explanations to be answers to why-questions.

${ }^{10}$ The connection to the production of mathematical knowledge is something that will be discussed more later in this paper.

${ }^{11}$ For a list of these different definitions see (Thurston $(1995)$, pg. 30)
} 
this question. Instead, given a particular circumstance, there is often a definition that stands out as best for that circumstance. For instance, when studying smooth manifolds it makes the most sense to use the definition stated in terms of tangent bundles and connections (Thurston (1995), definition 37). This does not necessarily solidify this definition's status as explanatory, but it does seem to make it a contender (at least to count as explanatory in this particular circumstance) ${ }^{12}$

I will now discuss a particular circumstance and consider which definition of the derivative is explanatory in that circumstance. I will focus on the question of which is the right definition of the derivative in the context of an introductory calculus class. A common way of introducing the derivative (at least in a pure mathematics class) is through the limit definition: ${ }^{13}$

$$
f^{\prime}(a)=\lim _{x \rightarrow a} \frac{f(x)-f(a)}{x-a}
$$

This definition is helpful because it clearly formalizes the geometric fact that the derivative at a point is equivalent to the slope of the tangent line at that point - a slope which is, in turn, the limit of the slopes of the secant lines 14 The function that we are taking the limit of is just the formula for the slope of the secant line through $f(x)$ and $f(a)$. So this is really the limit of the slopes of secant lines as the secant lines approach the tangent line of $f(a)$. By drawing out this corresponding limit, we can see the secant lines approximate the tangent line (see Figure 1). This illustration gives us a better grasp of what the derivative represents -

\footnotetext{
12 This is not something that I will discuss in depth during this paper, but I would like to point out that the notion of explanatory definition as I have presented it has not ruled out the possibility of a notion having more than one definition that counts as explanatory.

${ }^{13}$ Note that this definition can be generalized to give the derivative as a function, rather than the value at a specific point, but we will use the definition of the derivative at a point for simplicity.

${ }^{14}$ This exact limit definition does not appear on Thurston's list though it is equivalent to what he calls the logical definition, and clearly formalizes what he calls the geometric definition.
} 
so, it makes the concept of derivative more accessible to us. In this sense, the limit definition of the derivative is an explanatory definition.

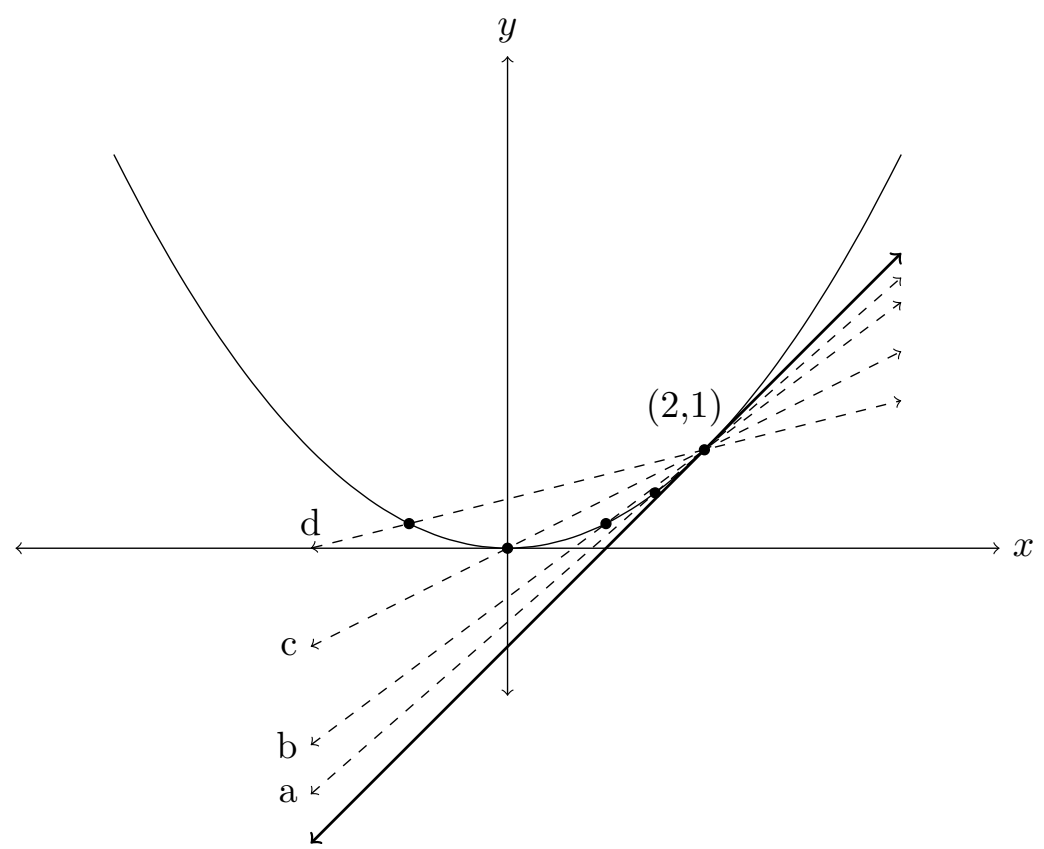

FIGURE 1. This figure shows the graph of $\frac{x^{2}}{4}$ (the solid curve), four secant lines (the dashed linear functions), and the tangent line at the point $(2,1)$ (the bold linear function). All of the secant lines pass through the point $(2,1)$, and so this illustrates that the limit of the secant lines approximates the tangent line at the point $(2,1)$. Thus, illustrating the limit definition of the derivative.

If you are introducing the concept of derivative to an unfamiliar audience, it is often very effective to draw an example graph and go through the illustrative calculations involved in computing the limit of the secant lines. This is effective because the illustration itself makes the derivative more accessible to the audience. They are able to see how the secant lines approximate the tangent, and they are able to grasp the concepts underlying the formal calculation of the derivative. 
Compare this definition to another, more algorithmic, definition. 15 This definition requires the memorization of an algorithm together with a few special cases. For instance, it defines the derivative of a polynomial with the following algorithm. Given a polynomial

$$
f(x)=a_{n} x^{n}+a_{n-1} x^{n-1}+\cdots+a_{1} x+a_{0}
$$

the derivative is

$$
f^{\prime}(x)=n \cdot a_{n} x^{n-1}+(n-1) \cdot a_{n-1} x^{n-2}+\cdots+a_{1} .
$$

This algorithmic presentation of the derivative can be learned by very young students, who might not even be familiar with the notion of slope. Such a student may be able to produce calculations of the derivative, but they will not have an understanding or appreciation for the deeper significance of this concept. This, unfortunately, is the result of most high school calculus classes, which emphasize the ability to compute the derivative rather than the underlying significance. Moreover, it has become common in general calculus classes - i.e., classes geared towards engineering and non-mathematics majors — to use this algorithmic definition rather than the more theoretical limit definition. This is because these non-mathematics fields are more interested in the ability to quickly and reliably compute derivatives than they are in having a deep understanding of what the derivative actually is.

\footnotetext{
${ }^{15}$ Thurston calls this the symbolic definition (Thurston (1995), pg. 30). It should be noted that this presentation of the derivative may be considered an algorithm suitable for certain cases (i.e., polynomials) and not a genuine definition. I do not want to discuss here whether or not we can legitimately classify it as a definition, but since it is often presented as a definition in introductory calculus classes at both the high school and college level, I will entertain it as such for this discussion. The purpose of this discussion is to highlight the values of the limit definition of derivative more than it is to highlight the failures of this algorithmic definition, and so taking this algorithm to be a definition does not harm my overall point.
} 
If the ultimate goal is only to compute rates of change and velocities for the purposes of application, then it may be argued that the algorithmic definition is all that is needed. But if this superficial definition is all that students are given, then they will not be aware of the connection between the derivative and the slope of the tangent line. This lack of awareness amounts to an inaccessibility of the deeper meaning of the derivative. The limit definition, however, makes this deeper meaning accessible, by explicitly presenting the definition as the limit of the slopes of the secant line (equivalently, the slope of the tangent line). The accessibility provided by this limit definition is what makes it explanatory. A student who learns about the derivative via this definition will be able to explain the connection between the derivative and the slope of a tangent line and will be familiar with the deeper meaning of the derivative.

In short, the limit definition of the derivative makes the concept of derivative more familiar to us. By considering the slopes of secant lines as they approach the relevant tangent line we get a better idea of what the derivative actually represents - i.e., the concept of derivative becomes more accessible to us. This accessibility makes the limit definition explanatory.

Now that we have seen an example of an explanatory definition, I would like to point out that explanatory definitions are pedagogically beneficial. The use of explanatory definitions to introduce concepts will result in more effective teaching. One concern with this pedagogical point is that there will be some students who do not benefit from an explanatory definition. For instance, there will be some students who take a class that presents the limit definition of the derivative who will not successfully grasp the concept of derivative. So one may object by saying that such students provide evidence that this definition is not explanatory. 
In response, notice that a definition can only be useful to a person when that person has the sufficient means for understanding the terms in which the definition is stated. In cases where students fail to benefit from the limit definition of the derivative, it is likely that the student has not adequately grasped limits, secant lines, or tangent lines. So the student does not have adequate conceptual means for grasping the limit definition. In these cases it is not a flaw in the definition, but rather a flaw in the students mathematical development.

The account of explanatory definition presented here is not meant to suggest that anyone who encounters an explanatory definition will appreciate the deep significance of the concept. Instead, the account grants that an explanatory definition will make the mathematical concept more accessible. The degree of accessibility depends on the qualifications of the person engaging with the definition. A trained mathematician will be fully equipped to access the concept of derivative after considering the limit definition. In the pedagogical case, the degree of accessibility of the concept will depend on the abilities of the student. For a top student who has followed along in class thus far, the limit definition will make the concept more accessible than it would for a student who has thus far struggled in class.

These degrees of accessibility correspond to a person's ability to improve or gain insight over time, after having encountered a definition on multiple occasions and in multiple contexts. For instance, a student who is taking calculus for the first time will not appreciate the depth of the concept of the derivative to the same extent as someone who has taken analysis, who in turn will not have the same extent of appreciation as someone who has taken differential geometry.

Explanatory definitions are not a fool-proof way of gaining access to mathematical concepts, but instead are a way of making more sense of these concepts. Exposure to explanatory 
definitions will increase the accessibility of the concept and this will allow a person to make more sense of the concept. But, a person will not be able to make perfect sense of a concept until they have developed the sufficient mathematical background.

So far I have given an account of explanatory definitions and demonstrated that such things exist by discussing an example. I have not yet discussed how this account of explanatory definitions fits into the existing literature on mathematical explanation, which has focused on cases of explanatory proof. I do not mean for my account of explanatory definition to take the place of explanatory proof, but instead intend for it to be considered in conjunction with explanatory proofs. I would now like discuss the difference between these two kinds of explanations.

The distinction between explanatory proofs and explanatory definitions is similar to a distinction mentioned by Silvia De Toffoli and Valeria Giardino in a paper about proofs in knot theory (De Toffoli \& Giardino (2016)). The goal of their paper is to demonstrate that when philosophy of mathematics focuses only on foundational questions, it neglects philosophically interesting questions arising from the analysis of mathematical practice. In particular, they point out that a purely foundational approach suggests that "philosophy of mathematics should not account for the production of mathematical knowledge but [that] its sole concern is its 'final' justification" (De Toffoli \& Giardino (2016), pg. 25). This distinction between "final justification" and the "production of mathematical knowledge" can be understood as the difference between knowing that a proof exists, and knowing how to actually carry out and produce a proof. Note that knowing a proof exists is sufficient for knowing that the theorem is true, but mathematicians often seek a deeper kind of knowledge. Namely, the kind of knowledge that 
allows them to produce proofs. The ability to produce a proof requires a certain degree of familiarity with the relevant mathematics - the relevant mathematics needs to be accessible to you — and this is precisely what we get from explanatory definitions.

Explanatory definitions contribute to the production of knowledge because they give mathematicians the means to grasp (and to reason about) abstract mathematical objects and constructions. They make mathematics more accessible so that conjectures can be formulated and mathematics can progress. Explanatory proofs, on the other hand, contribute primarily to the justification of mathematical knowledge. They explain why a theorem is true and so explain why we are justified in claiming knowledge of that theorem. To be fair, explanatory proofs do not solely play a justificatory role, but of course also play an explanatory role. So I don't want to claim that explanatory proofs are a lesser form of explanation than explana-

tory definitions, but rather, I want to emphasize that explanatory definitions contribute to the production of mathematical knowledge in a way that explanatory proofs do not. In general, explanatory proofs will not result in an increased accessibility of mathematical concepts. Whereas explanatory definitions enable us to better grasp mathematical concepts themselves, explanatory proofs only enable us to grasp the justification of mathematical facts.

\section{Explanatory Induction}

To show that there are instances of explanatory induction, I discuss the inductive construction of what algebraic topologists call " $C W$-complexes". The basic idea is of spaces that are constructed by attaching disks to one another. An $n$-disk (or $n$-dimensional disk) is just the 
space enclosed by and including the $(n-1)$-sphere $\sqrt{16}$ So, the 0 -disk is a point. The 1 -disk is the interval $[0,1]$. The 2 -disk is the set of points in the Euclidean plane that are of distance less than or equal to 1 from the origin, and so on.

To construct $C W$-complexes, we start with a set of 0-disks. We then attach disks of higher dimensions to these 0-disks using what are called "attaching maps". For instance, we can construct the unit circle, or the 1-sphere, by attaching a 1-disk to a 0-disk. We do so by taking the 1-disk, $[0,1]$, and mapping the endpoints, 0 and 1, to the 0 -disk (see Figure 2). This is essentially the same as taking a string and joining its ends together to form a closed loop one which can then be shaped into a circle.

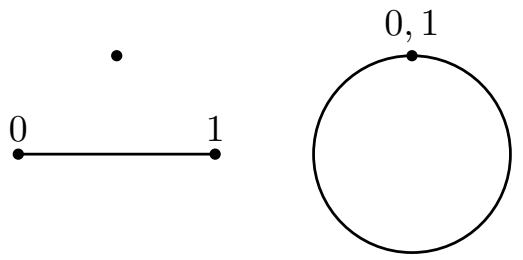

Figure 2. On the left we have a 0-disk and a 1-disk. On the right we have the result of mapping the endpoints of the 1-disk onto to the 0-disk, which consequently forms a 1-sphere or circle.

Similarly, we can use this technique to construct the 2-sphere - i.e., the surface of a ball. We do so by starting with one 0-disk and attaching to it one 2-disk. In particular, we identify the boundary - i.e., the unit circle surrounding the 2-disk - to the 0-disk. In doing so, we shrink the boundary to a point which causes the interior to "balloon out". See Figure 3.

In principle, we can construct any $n$-sphere in this way - i.e., by attaching an $n$-disk to a 0 -disk. With this example, we begin to see the intuitive idea of $C W$-complexes. Loosely,

\footnotetext{
${ }^{16}$ To be precise these $n$-disks consist only of the interior and do not include the boundary (i.e., the surrounding sphere). For simplicity, I define disks to include boundaries, which will simplify the discussion of attaching maps. For a more detailed and precise account of these $C W$-complexes see (Hatcher $(2001), \mathrm{Ch}$. 0).
} 

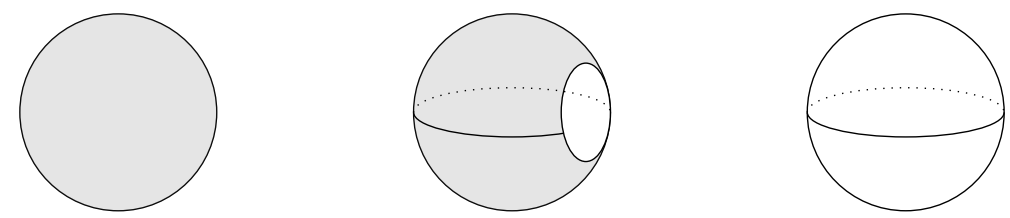

Figure 3. The above picture illustrates how the 2 -disk is attached to the 0 -disk to get the 2-sphere. The picture on the left is just the 2-disk. The middle depicts mapping the 2 -disk to the 0 -disk. So this picture shows a 3 -dimensional object, which is hollow and where the interior of the 2-disk on the left is beginning to form the surface of the 2 -sphere, i.e. the surface of a ball. Lastly, on the right, we have the 2 -sphere.

a topological space is a $C W$-complex if it can be constructed by attaching disks (of varying dimensions) to each other ${ }^{17}$ These $C W$-complexes are given by what is called a skeleton, $\left\{X^{n}\right\}_{n \in \mathbb{N}}$, which is defined by induction on the dimension of disks. $X^{0}$ is the discrete set consisting of the 0 -cells needed to construct the space. $X^{1}$ is the space resulting from attaching the 1-disks to $X^{0}, X^{2}$ is the space resulting from attaching the 2-disks to $X^{1}$, and so on ${ }^{18}$ So it is this inductively defined skeleton that gives the $C W$-construction ${ }^{19}$

Let me now be a bit more explicit about why the inductive construction of these $C W$ complexes should be considered explanatory. On the face of it, most topological spaces are difficult to grasp. For instance, the 3 -dimensional sphere, which sits in $\mathbb{R}^{4}$ and consists of all points of distance one from the origin is quite unfamiliar to us. We cannot seem to imagine or intuit it in its entirety. At best, we can imagine what the 3 -sphere is like locally. That is, given that it is a 3-manifold we know that at every point, it locally looks like an open subset of $\mathbb{R}^{3}$. This local picture, however, does not help us to intuit the space as a whole. Following

\footnotetext{
${ }^{17}$ More specifically the topological space must be a Hausdorff space - i.e., you must be able to separate any two distinct points with disjoint open sets.

${ }^{18} \mathrm{So}$, in general, $X^{n}$ is the space resulting from attaching the $n$-disks to $X^{n-1}$. Here by "attaching $n$-disks to $X^{n-1}$ ", I mean that $X^{n}$ is gotten by taking the disjoint union of $X^{n-1}$ and the set of $n$-disks. The attaching maps are used to give the equivalence relation needed for defining these disjoint unions.

${ }^{19}$ So a topological space that admits a $C W$-construction is called a $C W$-complex.
} 
the same procedure above, however, we know that its $C W$-construction is given by attaching a 3 -disk to a 0 -disk. The 3 -disk is simply the 2 -sphere including the interior - i.e., a solid ball. We are familiar with both a 3 -disk and a 0 -disk, and so the $C W$-construction allows us to put the 3-dimensional sphere in familiar terms, which in turns makes it more accessible to us. Moreover the attaching map for this $C W$-complex maps the boundary of the 3-disk, i.e., a 2-sphere, to a point. In other words the attaching map is essentially condensing a 2-sphere to a point, which is again something accessible and intuitive. With this $C W$-construction, the 3-sphere as a whole becomes more accessible to us.

Of course, there is still some mystery in this construction. In particular, the way that the interior of 3-disk will balloon out is not accessible in the way it was in the case of the 2-disk. It is difficult to imagine what it would look like for a solid 2-sphere - a solid ball — to "balloon out" into a 4-dimensional object. But this does not detract from my basic point: the $C W$-construction provides an illuminating definition of the 3 -sphere by presenting it to us in familiar terms.

This point becomes more salient the more complex our cases get. That is, $n$-spheres are one of the more accessible topological spaces because their standard presentation is fairly simple. But, $C W$-constructions build topological spaces out of these $n$-spheres. So, consider a less accessible topological space: $n$-dimensional real projective space, $\mathbb{R P}^{n}$. Intuitively, real projective space, $\mathbb{R} \mathbb{P}^{n}$, is the space consisting of lines in $\mathbb{R}^{n+1}$ passing through the origin. Topologically, $\mathbb{R} \mathbb{P}^{n}$ is taken to be the quotient space of $\mathbb{R}^{n+1} \backslash\{0\}$ under the equivalence relation $v \sim \lambda v$ for $v \in \mathbb{R}^{n+1}$ and $\lambda \in \mathbb{R} 20$

\footnotetext{
${ }^{20}$ For more details on this particular example, and other examples see (Hatcher (2001), pg. 6).
} 
A $C W$-construction of $\mathbb{R} \mathbb{P}^{n}$ consists of one $k$-disk for every $0 \leq k \leq n$. For instance, $\mathbb{R P}^{1}$ is constructed by attaching a 1-disk to $\mathbb{R P}^{0}$, which is just a point. More specifically, we can think of $\mathbb{R P}^{0}$ as the point at infinity, and the points of the 1-disk as encoding the slopes of the lines through the origin in $\mathbb{R}^{2}$. Thus, this attaching map yields $\mathbb{R} \mathbb{P}^{1}$. More generally, from the $C W$-construction we see for all $n \in \mathbb{N}$ how $\mathbb{R} \mathbb{P}^{n}$ is related to $\mathbb{R} \mathbb{P}^{n-1}$ - i.e., we get $\mathbb{R} \mathbb{P}^{n}$ by attaching an $n$-disk to $\mathbb{R} \mathbb{P}^{n-1}$. Since these $n$-disks are more accessible to us than projective space itself, the $C W$-construction makes projective space and its properties more accessible to us.

Recall that I formulated the notion of explanatory definition as follows:

Explanatory definition:: A mathematical definition is explanatory if it makes the mathematical concept being defined more accessible — i.e., it explains some feature or property of the relevant concept.

Above I gave a brief description of the way that $C W$-constructions can be used to define $n$-spheres and real projective space, but I have not yet discussed in detail how these $C W$ constructions make the spaces accessible. This will be the focus of the following paragraphs, but the general idea can be summarized as follows: the geometric and topological properties of a space are illuminated by their $C W$-constructions.

The fundamental idea behind $C W$-constructions is that these spaces can be built "step by step" by attaching disks of different dimensions. Each of these "steps" tells us something about the structure of the space - i.e., it tells us how the dimensions of the space relate to one another. A $C W$-construction builds up a space from disks of varying dimensions, but this building up can also be viewed as a decomposition — namely, a decomposition of a space 
into disks of varying dimension ${ }^{21}$ This decomposition reveals the geometric and topological properties of a space.

For instance, when a single point is removed from an $n$-sphere, the resulting space (i.e., "the punctured $n$-sphere") is homeomorphic to $\mathbb{R}^{n}$. This result is not obvious when we consider the $n$-sphere as the subset of $\mathbb{R}^{n+1}$ consisting of all points of distance exactly one from the origin. It does become obvious, however, when we consider the $C W$-construction of the $n$ sphere. For simplicity, consider again the 2-sphere, which is constructed from a 0-disk and a 2-disk by mapping the boundary of the 2-disk to the point as discussed above. So in this $C W$-construction the 0 -disk and the boundary of the 2-disk are all identified as the same point. Then removing a point from the 2-sphere amounts to removing the 0 -disk and the boundary of the 2-disk from the $C W$-construction. This leaves us with an open 2-disk - i.e., a 2-disk without its boundary. Further, the open 2-disk is topologically equivalent to $\mathbb{R}^{2}$ and so we have that the punctured 2-sphere is homeomorphic to $\mathbb{R}^{2}$. In general, the $C W$-construction of the $n$-sphere maps the boundary of a $n$-disk with a 0 -disk (i.e., a point). So from these $C W$-construction we can see that for every $n \in \mathbb{N}$, the punctured $n$-sphere is homeomorphic to $\mathbb{R}^{n}$.

The standard definition of the $n$-sphere - i.e., the space consisting of all points of exactly distance one from the origin - does not provide the geometric insight that this $C W$-construction does. The reason that the $C W$-construction reveals some geometric properties of a topological space is that it decomposes the space into its dimensional parts and the geometric relations

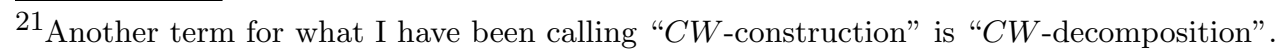


between these parts - i.e., $n$-disks and attaching maps. The fact that we are able to construct these topological spaces inductively, i.e., step-by-step, allows us to better understand the geometric relations of these different dimensions.

$C W$-constructions also make the topological properties of a space more accessible. For example, the homology of a space can be easily calculated from its $C W$-construction. Homology is one of the focuses of algebraic topology and essentially counts the number of $n$-dimensional holes a space has ${ }^{22}$ Briefly, the homology groups of a space are calculated from chain complexes, and forming these chain complexes is often the difficult part of computing homology groups $C W$-constructions, however, provide an easier way of forming a chain complex that can be used to compute homology groups. The $n$-disks used in a particular $C W$-construction yield a cellular chain complex. The homology groups of the space can then be calculated from this cellular chain complex. To summarize, the $n$-disks used in the $C W$-construction of a space form a chain complex from which the homology groups can easily be computed ${ }^{24}$ This improvement in computation is a direct result of the fact that a $C W$-construction decomposes a space into its dimensional parts - i.e., the $C W$-construction makes the homology of a space more accessible.

The above are just two examples of the ways that $C W$-constructions make the geometric and topological features of a space more accessible. In general, the fact that $C W$-constructions highlight the way that the dimensional parts of a space relate to one another is what allows

\footnotetext{
${ }^{22}$ Here I am limiting the discussion to singular and simplicial homology.

${ }^{23} \mathrm{~A}$ chain complex is given by

$$
\cdots \rightarrow G_{n+1} \stackrel{\partial_{n+1}}{\longrightarrow} G_{n} \stackrel{\partial_{n}}{\longrightarrow} G_{n-1} \stackrel{\partial_{n-1}}{\longrightarrow} \cdots \stackrel{\partial_{2}}{\longrightarrow} G_{1} \stackrel{\partial_{1}}{\longrightarrow} G_{0} \stackrel{\partial_{0}}{\longrightarrow} 0,
$$

where each $G_{i}$ is an abelian group, each $\partial_{i}$ is a group homomorphism, and for every $i \in \mathbb{N}, \partial_{i} \partial_{i+1}=0$.

${ }^{24}$ For more details and some examples, see (Hatcher (2001), pp. 137-146).
} 
for this geometric and topological insight. In this way, $C W$-constructions make topological spaces more accessible to us. These constructions take an abstract and seemingly unintuitable space and present it in such a way that the geometric and topological features of the space become apparent. Then, since a $C W$-construction can be seen as a way of defining a space and these constructions make the space more accessible, the $C W$-construction of a space is an example of an explanatory definition. Moreover, since these $C W$-constructions are inductive, they serve as an example of a way that mathematical induction is explanatory. This example diverges from the standard picture of mathematical explanation as consisting of explanatory proofs, but if we broaden our understanding of mathematical explanation then we get a better sense of how induction is explanatory.

\section{Conclusion}

I began this paper by mentioning Lange's argument against induction and commenting that it spurred many responses. The mere multitude of these adverse responses suggests that a number of people intuitively believe that induction should be considered explanatory, at least in some sense. In this paper, I have shown that there is a sense in which induction can be explanatory - namely, in the case of inductive definition. So one conclusion to draw from this paper is that there are in fact cases of explanatory induction in mathematics.

To argue for the above conclusion, however, it was necessary to broaden the account of mathematical explanation by introducing the notion of explanatory definition. The focus of the mathematical explanation literature had previously been on explanatory proof, but here I have argued that there are other forms of explanation within mathematics that require philosophical attention. In the above, I have provided two examples of explanatory definitions - the limit 
definition of the derivative and the $C W$-construction of topological spaces. These examples demonstrate not only that there is substance behind the notion of explanatory definition, but also how these definitions can contribute to mathematical progress and development. In advanced mathematics, it is common that the relevant mathematical objects will be unfamiliar, and initially inaccessible, to us, but explanatory definitions enable us to become familiar with such objects. In order to develop new conjectures and to progress within mathematics, it is necessary to develop a familiarity with these advanced mathematical concepts, which is precisely what explanatory definitions do.

Acknowledgments: I would like to thank both Curtis Franks and Tim Bays for their feedback on various drafts of this paper, and also the anonymous reviewers who provided numerous helpful comments on an earlier draft of this paper.

\section{REFERENCES}

Arnauld, A. \& Nicole, P. (1683). La Logique ou L'art du Penser. Translated by J. Buroker as Logic, or the Art of Thinking, (1996). Cambridge: Cambridge University Press.

Baker, A. (2010). Mathematical Induction. Analysis, 70(4), 681-689.

Baldwin, J. (2016). Foundations of Mathematics: Reliability AND Clarity: the explanatory role of mathematical induction. In J. Väänänen, A. Hirvonen, \& R. de Queiroz (Eds.), 
Logic, Language, Information, and Computation: 23rd International Workshop, WoLLIC 2016, Puebla, Mexico, August 16-19th, 2016. Proceedings (pp. 68-82). Berlin: Springer.

Baldwin, J. (2017). The explanatory power of a new proof: Henkin's completeness proof. In M. Piazza \& G. Pulcini (Eds.), Truth, Existence, and Explanation: FilMat 2016 Studies in Philosophy of Mathematics (Forthcoming). Springer.

De Toffoli, S. \& Giardino, V. (2016). Envisioning Transformations - The Practice of Topology. In B. Larvor (Ed.), Mathematical Cultures (pp. 25-50). Zurich: Springer.

Giaquinto, M. (2008). Cognition of Structure. In P. Mancosu (Ed.), Philosophy of Mathematical Practice (pp. 43-64). New York: Oxford University Press.

Hafner, J. \& Mancosu, P. (2005). The Varieties of Mathematical Explanation. In P. Mancosu et al. (Eds.), Visualization, Explanation and Reasoning Styles in Mathematics (pp. 215-250). Dordrecht ; Norwell, MA: Springer.

Hatcher, A. (2001). Algebraic Topology. Cambridge ; New York: Cambridge University Press. Hoeltje, M., Schiender,B., \& Steinberg, A. (2013). Explanation by induction?. Synthese, 190(3), 509-524.

Lange, M. (2009). Why proofs by mathematical induction are generally not explanatory. Analysis, 69(2), 203-211.

Lange, M. (2017). Because Without Cause. New York: Oxford University Press.

Tappenden, J. (2008). Mathematical Concepts and Definitions. In P. Mancosu (Ed.), Philosophy of Mathematical Practice (pp. 256-275). New York: Oxford University Press.

Tappenden, J. (2008). Mathematical Concepts: Fruitfulness and Naturalness. In P. Mancosu (Ed.), Philosophy of Mathematical Practice (pp. 276-301). New York: Oxford University 
Press.

Thurston, W. (1995). Proof and Progress in Mathematics. For the Learning of Mathematics, 15(1), 29-37. 